\title{
Predictors of Group Cognitive Behaviour Therapy outcomes for the treatment of depression in Malaysia.
}

\begin{abstract}
The aim of this study was to identify predictors of response to treatment for depression in Malaysia, using demographic and cognitive predictors. 113 patients, that were diagnosed with depression, were randomly assigned to the Treatment-As-Usual (TAU) $(n=55)$, or TAU plus eight sessions of Group Cognitive Behaviour Therapy (TAU + GCBT; $n=58$ ). Pre-treatment using the Beck Hopelessness Scale (BHS), the Automatic Thoughts Questionnaire-Malay (ATQ-Malay), the Dysfunctional Attitude Scale-Malay (DAS-Malay), a quality of life scale, and demographic characteristics, were used in a series of multiple regression models, as potential predictors of the Beck Depression Inventory-Malay (BDI-Malay) post-assessment scores. Regression results revealed that age, the quality of life scale, and all three cognitive measures were significant predictors of outcomes in the Group Cognitive Behaviour Therapy (GCBT) group, showing that Beck's cognitive model for depression could be applied in Malaysia.
\end{abstract}

Keyword: Group CBT; Depression; Predictors; Treatment outcome; Malaysia. 